\title{
Justification of the Technology for Preventing Scale in the Downhole Equipment
}

\author{
LiliyaAl'bertovnaShangaraeva ${ }^{1}$ \\ ${ }^{1}$ National mineral resources university "University of Mines", Russia \\ Correspondence: LiliyaAl'bertovnaShangaraeva, National mineral resources university "University of Mines“, \\ 21st line, 2, St-Petersburg, 199106, Russia.
}

Received: January 15, 2015

Accepted: February 13, $2015 \quad$ Online Published: July 25, 2015

doi:10.5539/mas.v9n8p270

URL: http://dx.doi.org/10.5539/mas.v9n8p270

\begin{abstract}
The formation of scale in the downhole equipment and the bottomhole formation zoneis often a serious cause of energy losses, it reduce the productivity of the wells, leading to the current unplanned and costly workovers. Fight with scale in the well operation is one of the most important ways to increase the efficiency of oil production. The technology for preventing scale in the downhole equipment was investigated in the present work.
\end{abstract}

Keywords: Scale, downhole equipment, inhibitor, formation squeeze treatment, phosphonic acid, bifluoride of ammonia, bottomhole formation zone, adsorption-desorption characteristics

\section{Introduction}

One of the major problems in the oil and gas industry is to enhance oil recovery in complicated geological and technological conditions. This is due to the assumption of the most of high-yield oil fields in the final stage of the development and the growing proportion of hard to recover reserves to their total amount. Nowadays process of oil extraction is significantly complicates with high water cut production that leads to scale in the downhole equipment (Muslimov, R., 2005)

An integral part of the development and exploration of oil fields is a water factor. As a result of flooding is the formation of scale. Accumulation of scale in the downhole equipment leads to reduced productivity, premature failure of the pumping equipment, current unscheduled and costly workovers and as a consequence, a significant deterioration of the technical and economic indices of oil and gas companies (Zdolnik, S., \&Akimov, O., 2009).

Water is of paramount importance in the oilfield, as the scale will occur only when the water is used. Water is a good solvent for many materials and can carry large amounts of scale mineral.

All natural water contains dissolved components resulting from contact with the mineral phases in the natural environment.This leads to the fact that the complex fluids, which are rich in ions, some of which are at the limit of saturation for certain mineral phase, is form (Krabrtri, M., et al., 2002).

Calcium carbonate, calcium and barium sulfate are major components of most oilfield scale. In the oil wells deposits of pure scale are rare. Typically they are a mixture of one or more basic inorganic components with corrosion products, sediments and particles of sand, or covered with asphalt resins paraffinic substances. Wells treatment cannot be successful without the removal of the organic component of the scale (Zdolnik, S., \& Akimov, O., 2009).

The mechanism of scale formation consists of several stages. The first stage of development begins with a saturated solution of unstable formation of clusters of atoms. Then formed primary crystal nucleus, when atomic clusters transform into small crystals-buds. These crystals gradually grow due to adsorption of ions on the surface of the crystal imperfections, increasing their size, are combined together into larger aggregates. For some time large crystals formed in the solution or aggregates that they may no longer be kept in suspension in the solution is released and the solid phase (precipitate) (Krabrtri, M., et al., 2002).

Crystal growth occurs at the initiation of certain physico-chemical reactions at the existing boundary between a solid and a liquid. Portions of such reactions are various surface defects such as roughness surfaces of the tubes, the perforations, joints and seams in tubing and pipelines (Kashchavtsev, V., 2005). 
Scale occur in three forms: as a thin scale or friable flakes in a layered form, in crystalline form. The deposits of the first type have a loose structure, permeable and can be easily removed.

Radioactive barium scale are the most hard-to-out of scale. Barium is often found in highly mineralized formation waters of oil fields, where its concentration often reaches 0,15-0,5 gpl. Even in the presence of low concentrations of sulfate ion barite $\left(\mathrm{BaSO}_{4}\right)$ precipitates. These scale are less soluble. For example, in distilled water at $25^{\circ} \mathrm{C}$ dissolves all $0,0023 \mathrm{gpl}$ of barite, which is almost 900 times less than the solubility of gypsum (Antipin, Y., \&Islanova, G., 1999, Rizkalla E. 1983).

Noted that the barite scale, selected from oil-field equipment and tubing have increased radioactivity due to the presence of radioactive isotopes of radium, which are associated in groundwater with barium. Effect of pressure on the solubility of $\mathrm{BaSO}_{4}$ understudied. In general, there is a slight increase in solubility with increasing pressure is particularly pronounced in solutions with salinity less than 30-50 gpl. With the increase in salinity influence of pressure effect is negligible (Symeopoulos B. \&Koutsoukos P., 1999). Thermobaric conditions while moving upward flow of liquid through the wellbore produce little effect on the change in water solubility of barite.

The main reasons for scale in the wells are mixing incompatible waters resulting from the operation of several productive layers simultaneously or in wells operated with one layer with behind-the-casing flows from related horizons (Larichev T. et. al., 2006). Often the cause is a breakdown of the technical state of production casing and packer leaks, especially in fields on the later stages.

\section{Method}

The scale inhibition in downhole equipment does not have a unique solution, every case of scale formation has its own individual approach. Predicting possible complications associated with scale has particular importance in addressing such a complex multisided problem. For the successful solution requires constant well monitoring and monitoring of physico-chemical processes in it. Great help in this matter could have a map of changes in the produced water composition in various components of: chlorides, sulfates, barites and mineralization.

Casing leak of the production string that leads to scale formation in the wells can be detected as the resultbased onfollowing main characteristics. The main idea is to track changes in the produced water composition in the dynamics. So reducing the content of barium ions, then the appearance of sulfates, after complete disappearance of the barium shows may indicate leakage of production casing and imminent failure of the pump due to scale. An additional feature is the presence of barium sulphate in the suspended solids.

For most wells, was confined to Permian strata, is typical the content of barium ion in the range of $0,1-0,8 \mathrm{gpl}$, and a low content of sulfate ions in the range of $0-0,25$ gpl. Well under the influence of freshwater or wastewater downloadscontains thebarium ions in the range of 0 to $0,1 \mathrm{gpl}$. For waters of the upper horizons (except Tournaisian) is characterized by a high content of sulfate ions (from 1 to 12 gpl). If produced water from the Devonian wells has a sulfate ion concentration greater than $0,3 \mathrm{gpl}$, this wells require additional study in case of casing leak of the production string. The high content of sulfate ions without leakage may be the result of the activities of the enhanced oil recovery or geological and engineering operations (Shangareeva, L. \&Petukhov, 2013, Shangareeva, L. \&Petukhov, 2011).

Nowdays the most widespread in oil-field practice is the reagent method of preventing scaling. The wide range of chemical compounds which show the effective inhibiting properties against scale (Tomson, N. et. al., 2003, Glushenko, V. et. al., 2008). But, despite the huge contribution of scientists in the development of this direction, the development of chemical compositions remains a challenge (Guskova, I., 2011).

One of the most effective method is the injection of an aqueous solution of the scale inhibitor in the bottomhole formation zone for its subsequent long enough removal in the wellbore during its operation (Shaidakov, V., 2012). Existing methods of selection reagent for specific conditions are based on the selection of the brand of the inhibitor and on its capacity. The adsorption-desorption characteristics of the inhibitor are not taken into account. However, the amount of inhibitors adsorption on the formation and its subsequent desorption depends on it. They affect the efficiency and duration of effect of the compositions. At the same time, it is known that reservoirs have different wettability and different absorption capacity. In this regard, inhibiting composition has to meet strict requirements. It should adsorb as quickly and broadly as possible on the surface of the formation during injection, and should very slowly desorb from formations during well operation. Thus, the longer will be the process of removal of the reagent, the longer and more effectively will be preventing the scale. Selection of the scale inhibitor, taking into account its adsorption and desorption ability will provide the lowest removal reagent and increase the time and efficiency to prevent the scale (Ibragimov, N. et al., 2003). 
As an inhibitor of the growth of salt crystals is widely known use of phosphonic acids. However, an aqueous solution of these acids has a low adsorption-desorption ability and quickly taken out from the reservoir by fluid flow (Yarkeeva, N., 2008).

To improve the sorption properties of the inhibitors recommended their injection with weak acid solutions (Gimatutdinov, S., 1983). So treatment of the bottomhole formation zone is carried out by the mixture of phosphonic acids with hydrochloric acid. Due to the acidic reagent there is rejection of oil film from the surface of the reservoir by reducing the interfacial tension in the system "formation-oil-inhibiting solution". Hydrochloric acid reacts with carbonate minerals and thus the adsorption area of the inhibitor on reservoir increases.

The major volume of oil is produced from sandstone reservoirs with carbonate concretion. Therefore, scale inhibitor should reduce the surface tension at the boundary of the "formation-inhibitor solution" and react with both silicate, aluminosilicate and carbonate concretions. It enhances the adsorption-desorption properties of the inhibitor.

Composition, which contains phosphonicand hydrofluoric acids and surfactants (Antipin Y. et. al., 1993) is known. Hydrofluoric acid in the composition interacts with silicate and aluminosilicate minerals. But this acid is expensive and dangerous to handle. So it will be more sophisticated to use a composition containing bifluoride of ammonia and hydrochloric acid, which form hydrofluoric acid as a result of reaction. In addition, if the rock contains carbonates of calcium and magnesium, they can form insoluble fluorides of calcium and magnesium during their reaction with fluoric acid. Appending a hydrochloric acid to the composition will prevent this. In laboratory was developed effective composition that meets these requirements.

Inhibitory composition development was carried out in laboratory conditions at three stages. The first was selection the most effective combination of components as a result of determining the interfacial tension at the boundary "oil - inhibitor solution". Then the inhibiting ability of the composition is studied. The third step was determination the adsorption-desorption properties of scale inhibitor. Evaluation of the adsorption and desorption ability of inhibitor compositions were performed when filtering inhibiting compositions on the FDES-645 (Formation damage evaluation system). The developed composition represents multicomponent structure for receiving the greatest inhibiting action in relation to inorganic salts.

\section{Results}

Analyzing the obtained data, it is possible to note that increase of concentration of reagents reduces the size of aninterfacial tension, and hydrochloric acid has a greater influence on it. The optimal concentrations are $0,5-2 \%$ of a bifluoride of ammoniaand $7-10 \%$ of hydrochloric acid. Addition ofphosphonicacid to the compositioncan further reducetheinterfacial tension. This effect is noticeable when the content of acid in the amount of $3 \%$.

The assessment of efficiency of the inhibitors by the ability to prevent the precipitation was carried out according to the NACE standard TM 0374-2007 in the liquid solution of the averaged model of the formation water. Experiment results revealed that the developed chemical compositions have the necessary protective effect.

The use of chemicals to prevent scale in wells associated with the use of chemically aggressive fluids. Injection of corrosive agents in the bottomhole formation zone not only promotes downhole equipment failure, but also brings additional harm from the products which are formed as a result of exchange reactions and can worse the filtration properties of the bottomhole formation zone. All this leads to a significant increase in operating costs of oil and gas companies that have a negative impact on the oil production cost. Therefore it is necessary to consider the corrosiveness of injected compositions.

All considered chemical compositions showed acceptable corrosion rate in relation to the steel and these reagents can be considered as reagents to prevent scaling in the wells.

To determine the adsorption-desorption characteristics of inhibitory compositions was carried out filtration tests on core samples.

In the laboratory were studied three inhibitory compositions. Considering that the initial concentration of organophosphorus reagents in inhibiting compositions are different, it's possible to compare the dynamics of the relative concentrations of the solutions. While the relative concentration is determined by the ratio of their current concentration to the original.

Figure 1 shows the results of relative concentration of organophosphorus reagents determinationin the inhibiting compositions when the adsorption process.Laboratory studies have shown that the maximum adsorption is achieved by injection the nine pore volumes for inhibiting compositions \# 1 and \# 2, and ten pore volumes for 
composition \# 3.Adsorption proceeds faster when applying the inhibiting compositions \# 1 and \# 2.

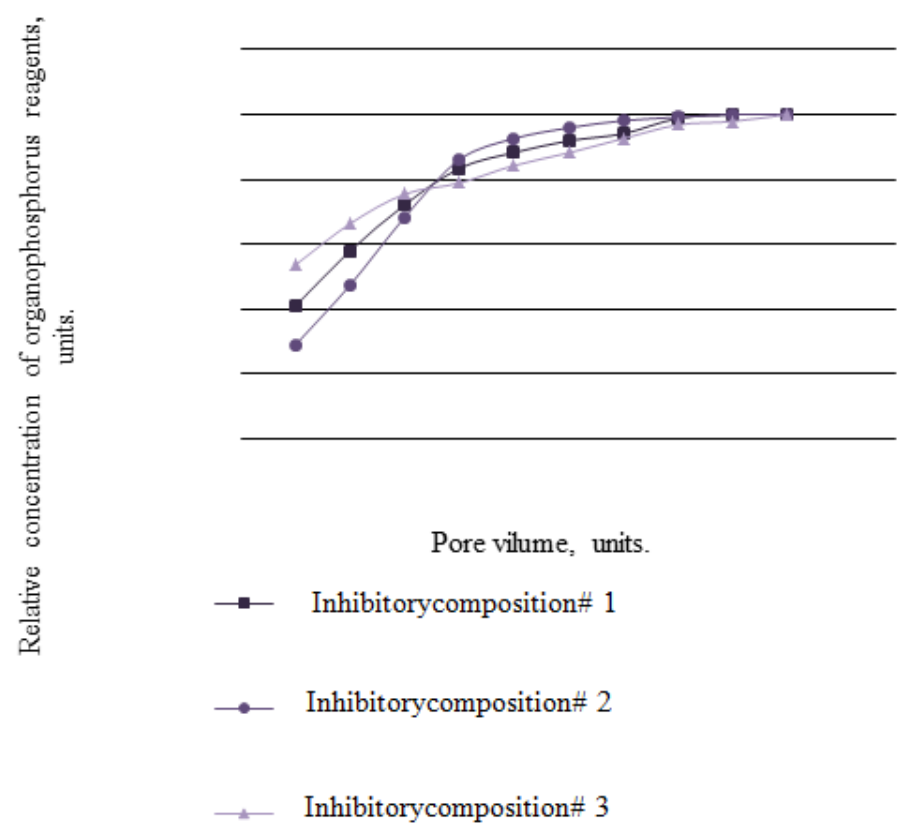

Figure 1. Changing relative concentration of organophosphorus reagentsin the process of adsorption

After the core was left for 24 hours through it was injected formation water to displace inhibitory composition. Figures 2 and 3 show the results of the relative concentrations of organophosphorus inhibitors determination during desorption.

According to figures 2 when using inhibitory composition \# 3 necessary subtraction of inhibitor, which is sufficient for effective protection against scale, is achieved by injection through a core sample 30 pore volumes of water. When using the inhibitory composition \# 1 effective protection against scaleis achieved by injection 46 pore volumes of water, and composition \# 2 - 42 pore volumes of water.

Analysis of data shows that significant portion of the free (not adsorbed) inhibitor is taken out when injection the first two pore volumes. Considering, however, that in the composition \# 1 is used concentration of organophosphorus reagents higher than that the composition \#2, the losses will be much more when applying the composition \# 1 . 


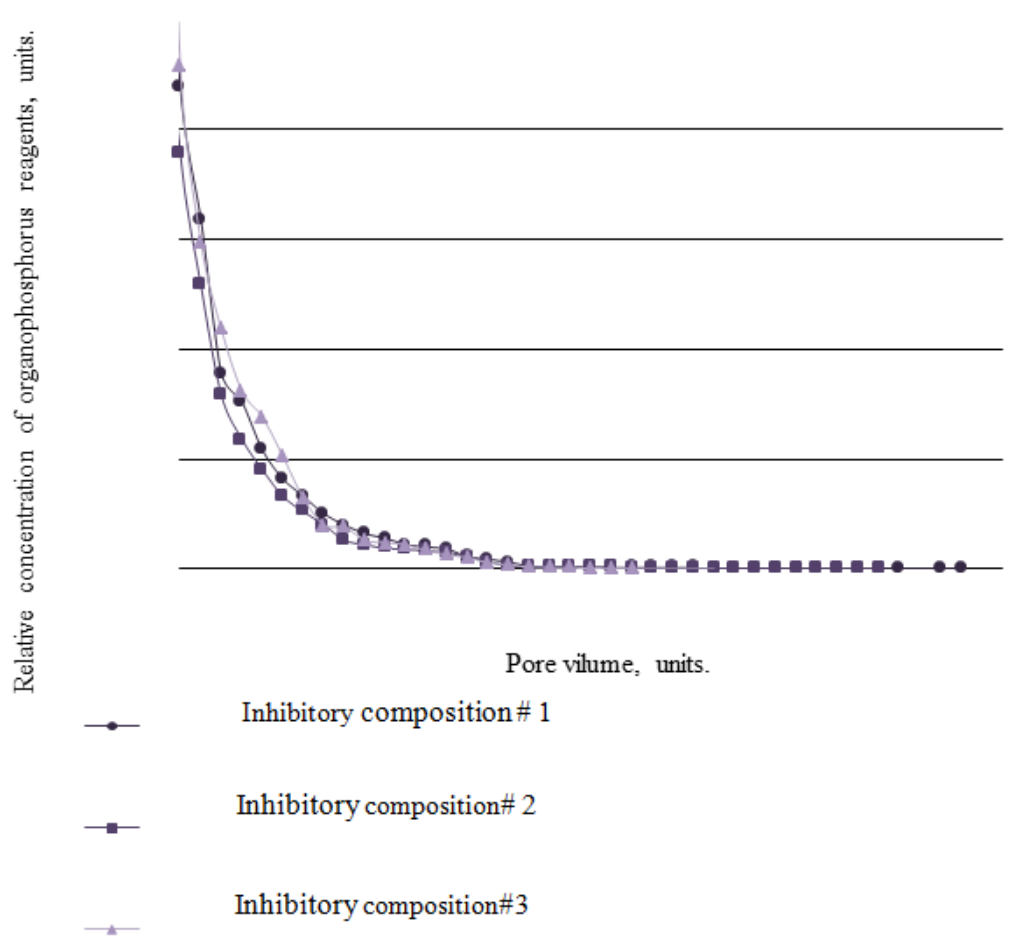

Figure 2. Changing relative concentration of organophosphorus reagents in the process of desorption

\section{Discussion}

Efficiency of the developed composition is explained by the mechanism of influence on the reservoir of acid additives included in the composition.

The fluoric acid which is formed as a result of interaction of hydrochloric acid with bifluoride of ammonia reacts with the quartz and kaolin which are a part of terrigenous collectors according to the following scheme:

$$
\begin{gathered}
\mathrm{SiO}_{2}+4 \mathrm{HF}=\mathrm{SiF}_{4}+2 \mathrm{H}_{2} \mathrm{O}, \\
\mathrm{H}_{4} \mathrm{Al}_{2} \mathrm{Si}_{2} \mathrm{O}_{9}+14 \mathrm{HF}=2 \mathrm{AlF}_{3}+2 \mathrm{SiF}_{4}+9 \mathrm{H}_{2} \mathrm{O} .
\end{gathered}
$$

Further there is an interaction of the formed fluoric $\mathrm{SiF} 4$ silicon to water:

$$
2 \mathrm{SiF}_{4}+4 \mathrm{H}_{2} \mathrm{O}=\mathrm{Si}(\mathrm{OH})_{4}+2 \mathrm{H}_{2} \mathrm{SiF}_{6} \text {. }
$$

Hydrofluosilicic acid formed as a result of reaction remains in solution and the hydrate of silicon oxide can turn into a gel in process of decrease in acidity of solution (Amiri M. \&Moghadasi J., 2010). The turned-out gel in turn can very easily block pores, thereby reducing the permeability of the reservoir. Mix of fluoric and hydrochloric acids is usedto prevent this when treatment of terrigenous reservoirs. Hydrochloric acid is necessary to maintain the high $\mathrm{pH}$ environment and retention of silicic acid in solution (Petrukhin O., 1992). Besides, if reservoir contains carbonates of calcium and magnesium during their reaction with fluoric acid almost hardly soluble fluorides of calcium and magnesium are formed. It is interfered by hydrochloric acid.

Also hydrochloric acid is capable to change wettability of reservoir, clearing its surface of the oil film. This achieves a smooth and more complete adsorption of the scale inhibitor.

Due to the attack by fluoric acid of a silicate reservoir surface the increase area of it surface and the extent of chemical composition adsorption on this surface is provided. The inhibiting composition will get into structure of silica sols, which cover the contact surface of the inhibitor with silicate minerals. Due to this it will desorb slower from the reservoir, as films of silica sols being a part of inhibitor composition are more resistant to leaching. Which leads to the fact that the time of desorption raises, and from this we can conclude that effectiveness of the scale inhibitor increases.

\section{Conclusions}

Thus, application of the developed inhibitor allows to increase desorption from formation in 1.6 times compared 
with the analogues. Wide range of effectiveness of the examined compounds is explained by the different mechanism of action on the formation of specific additives. Laboratory experiments have shown that desorption from the formation with the developed inhibitory compositions longer than the existing counterparts.

Besides development of the inhibitor compositions technological ways of their injection in a well are of great importance for the prevention of scale. One of the most effective methods is the intermittent injection of the chemical composition in the bottomhole formation zone.

According to the general principles of inhibitory protection of the downhole equipment and productive formation a preparatory work is necessary just before the implementation of the technology of the prevention of scale in the chosen well.

Originally manufactured sampling of products produced from the well to determine the degree of over-saturation ions sulfate and barium. Chemical analysis of water is carried out in the laboratory. To determine the productivity index is carried out pressure transient analysis. Existence of scale and their settling zone in a well are established by calipering. Operation of bottomhole cleaning, borehole cleaning, bottomhole treatment, cleaning of downhole equipment with an organic solvent in case of scaling and decreasing the productivity index.

Formation squeeze treatment of inhibitory composition is most preferable, as it can adsorbed in the bottomhole formation zone and held on the surface of the reservoir. In the process of fluid filtration through the bottomhole formation zone gradual desorption process proceeds, the scale inhibitor is released and e comes to a well with the rformation fluid, providing conditions to prevent scale.

Before carrying out the operation of formation squeeze treatment the candidate wells is selected. Candidate wells have to meet the following main criteria:

- the well is complicated by scale;

- the low mean time to failure of downhole equipment;

- the formation of scale below the pump suction and, therefore, of traditional methods of protection against scaling;

- the high level of solids.

To prepare the surface of the reservoir, remove already formed scale it is recommended to combine formation squeeze treatment with a small volume of acid treatment of wells. This technique, at the same time, allows to increase the permeability of the bottomhole formation zone and facilitates delivery of the inhibitor into the formation.

The advantages of the technology include the fact that the protection extends to the bottomhole formation zone of the well, the production casing to the level of the pump, downhole equipment, tubing and terrestrial communications. Another advantage is the length of the period of protection between treatments and no need for construction and operation of the dosers.

\section{References}

Amiri, M., \& Moghadasi, J. (2010). Prediction the amount of barium sulfate scale formation in Siri oilfield using OLI scaleChem Software. Asian Journal of Scientific Research ed., 3, 230-239. http://dx.doi.org/10.3923/ajsr.2010.230.239

Antipin, Y., \& Islanova, G. (1999). Use gel-forming technologies for increase of scale prevention efficiency in the wells. Development and Exploration of Oil and Gas Fields ed., pp. 67-77. Ufa: Ufimsky state oil technical university.

Gimatutdinov, S. (1983). Reference guide on design of development and exploration of oil fields. Oil production (p. 455). Moskow: Nedra.

Glushenko, V., Silin, M., \& Ptashko, O. (2008). Oilfield chemicals: Complications in the system reservoir - well - OPPU (p. 328). Moskow: Max Press.

Guskova, I. A. (2011). Design and development of technological solutions to the problem formation of organic deposits in the changed conditions of technogenic deposits of oil (Doctoral dissertation, Tatnipineft, Bugulma, Republic of Tatarstan, Russia).

Ibragimov, N., Khafizov, A., \& Shaydakov, V. (2003). Complications in oil production (p. 254). Ufa: Monograph. 
Kashchavtsev, V. (2005). Role of interstitial waters in the process of precipitation of scale during oil production. Oil, Gas and Business ed., 42-45.

Krabrtri, M., Eslinger, D., \& Fletcher, F. (2002). Fighting scale - removal and prevention. Oilfield review ed., 52-73.

Larichev, T., Sotnikova, L., \& Sechkarev B. (2006). Bulk crystallization in inorganic systems. (p.364). Kemerovo: Kemerovo State University.

Muslimov, R. (2005). Up-to-date methods of enhanced oil recovery. Design, optimization and evaluation of the performance (p. 205). Kazan: Academy of Sciences RT.

Patent. 1804469 (RF) Composition for preventing scale / Y.V. Antipin, A.Sh. Husniyarov, G.A. Shamaev: UGNTU. No. 4878526/03; published 23.03.93.

Petrukhin, O. (1992). Analytical Chemistry. Chemical methods of analysis. (p. 489). Moskow: Khimiya.

Rizkalla, E. (1983). Kinetics of Crystallisation of barium sulphate. J. Chem. Soc. Faraday Trans ed., 79, 1857-1867.

Shaidakov, V. (2012). About the dosing of chemicals into the well. Oil. Gas. Novation ed., 11, 39-42.

Shangareeva, L., \& Petukhov, A. (2011). Study of the deposition of salts in downhole equipment and the prediction of their education (Proceedings of the Conference. Rassohinskie Reading. for Graduate Students, 112-115. Uhta: Uhtinsky state technical university.

Shangareeva, L., \& Petukhov, A. (2013). Prediction of formation of scale in oil wells. Modern Problems of Science and Education. Retrieved from http://science-education.ru/113-11180

Symeopoulos, B., \& Koutsoukos, P. (1999). Radioanalytical monitoring of the formation of barium sulfate in aqueous solutions. Journal of Radoanalytlcal and Nuclear Chemistry ed., 173(1), 23-36.

Tomson, N., Watson, M., \& Fu, G. (2003). Mechanisms of Mineral Scale Inhibition, 18, 192-199. SPEPF. http://dx.doi.org/10.2118/84958-PA

Yarkeeva, N. R. (2008). Improving the efficiency of prevention of scaling in wells at a late stage of development of deposits. (PhD thesis, Ufimsky state oil technical university, Ufa, Russia).

Zdolnik, S., \& Akimov, O. (2009). Manage scaling - a pledge improve the efficiency of oil production Engineering Practice ed., Pilot, 66-69.

\section{Copyrights}

Copyright for this article is retained by the author(s), with first publication rights granted to the journal.

This is an open-access article distributed under the terms and conditions of the Creative Commons Attribution license (http://creativecommons.org/licenses/by/3.0/). 